\title{
POTENCIAL DO USO DA BELDROEGA NA SEGURANÇA ALIMENTAR DE COMUNIDADES EM SITUAÇÃO DE RISCO E VULNERABILIDADE SOCIAL
}

\section{POTENTIAL OF THE USE OF PURSLANE IN FOOD SECURITY OF COMMUNITIES AT RISK AND SOCIAL VULNERABILITY}

\author{
Raimeyre Nobre Dias ${ }^{1}$ \\ Terezinha Paula S. da Sila ${ }^{2}$ \\ Sandoval Menezes Matos ${ }^{2}$ \\ Dieny Michelly S. da Silva ${ }^{2}$ \\ Edgley Soares Silva ${ }^{3}$ \\ Célida Socorro Vieira dos Santos ${ }^{4}$ \\ Maria Fernanda Belingieri Durigan ${ }^{5}$
}

\begin{abstract}
RESUMO: O potencial da beldroega (Portulaca oleracea) tem chamado atenção de extensionistas, técnicos e pesquisadores devido sua rusticidade, adaptabilidade, podendo ser, alternativa para diversificar e melhorar a qualidade nutricional da dieta de populações de baixa renda. Esta pesquisa bibliográfica teve como objetivo socializar as principais potencialidades de uso da Portulaca oleracea que possam promover a segurança alimentar em comunidade em situação de risco e vulnerabilidade social. Elaborou-se pesquisa bibliográfica nas plataformas da Scielo, Google Acadêmico e periódicos da CAPES, com as palavras-chave: segurança alimentar; PANCS; Portulaca oleracea; comunidade em situação de risco e vulnerabilidade social, beldroega. A partir desta pesquisa, fica clara a potencialidade de uso de Portulaca oleracea na promoção da segurança alimentar em comunidades em situação de risco e vulnerabilidade social.
\end{abstract}

Palavras-chave: beldroega, PANCS, soberania alimentar

ABSTRACT: (Portulaca oleracea) has attracted attention of extensionists, technicians and researchers due to their rusticity, adaptability and low cost of production, being able to

\footnotetext{
${ }^{1}$ Mestranda em Agroecologia do Programa de Pós-graduação em Agroecologia (bolsista CAPES), Universidade Estadual de Roraima, e-mail: raimeyrenobre@ hotmail.com.

${ }^{2}$ Aluno(a) do curso de mestrado em Agroecologia - Universidade Estadual de Roraima - Programa de Pós Graduação em Agroecologia.

${ }^{3}$ Professor do curso de agronomia da Faculdade Roraimense de Ensino Superior-FARES.

${ }^{4}$ Doutora em solos e nutrição de plantas - Professora do Curso de Agronomia - Universidade Federal de Roraima-Campus Cauamé, Boa Vista-RR, 60310-000 celida.vieira@ufrr.br

${ }^{5}$ Doutora em Agronomia (Produção Vegetal), Orientadora, Pesquisadora da Empresa Brasileira de Pesquisa Agropecuária (Embrapa Roraima), e-mail: maria.durigan@embrapa.br.
} 
diversify and improve the nutritional quality of the diet of low income populations. This bibliographic research aimed to socialize the main potential of use of Portulaca oleracea that can promote food security in a community at risk and social vulnerability. Bibliographic research was carried out on the platforms of Scielo, Google Academic and CAPES journals, with the following keywords: food security; PANCS; Portulaca oleracea; community at risk and social vulnerability, prostitute. From this research, it is clear the potential use of Portulaca oleracea in promoting food security in communities at risk and social vulnerability.

Key words: burgher, unconventional food plants, PANCS, food overeignty.

\section{INTRODUÇÃO}

O Brasil tem uma grande biodiversidade de plantas ricas em nutrientes e minerais, plantas essas rusticas, adaptadas as condições locais, muitas consideradas daninhas, pragas na agricultura convencional. As hortaliças não convencionais são alternativa alimentar e uma opção de atividade agropecuária (DIAS et al., 2005), podendo gerar trabalho, emprego e renda, além de serem plantas com excelente valor nutricional, de fácil cultivo e baixo custo (ROCHA et al., 2008).

As deficiências nutricionais são um dos grandes problemas no mundo e representam grande desafio para a saúde pública no Brasil, a falta de alimentos em quantidade e qualidade nutricional e uma realidade nas comunidade em situação de risco e vulnerabilidade social. A falta de hábitos saudáveis é uma realidade presente na sociedade, a dificuldade de diversificar a alimentação é um desafio que devemos focar para garantir uma promoção de alimentação saudável.

As plantas alimentícias não convencionais caracterizam-se como alternativa para diversificar e melhorar a qualidade nutricional da dieta de populações de baixa renda, pois, podem ser de fácil cultivo, resistentes, boa produtividade e, podem ser cultivados em seus domicílios ou em pequenas hortas comunitárias.

Segundo Gallina e Melão (2012) a segurança alimentar deve ser garantida a toda população, isto significa acesso de forma continuada a alimentos que sejam seguros e tenham qualidades, em quantidades suficientes para suprir suas necessidades, sem comprometer o acesso a outras demandas. Os alimentos devem ser seguros, nutritivos e saborosos, para que o mesmo possa ter condições de desenvolvimento físico e mental saudáveis. É importante ressaltar que as práticas alimentares que promovem a saúde, devem respeitar à diversidade cultural, social, econômica e ambientalmente sustentável.

Nesse sentido, a Portulaca oleracea L (Beldroega), pertencente a família Portulacaceae, conhecida como erva daninhas anual, tem chamado atenção devido sua rusticidade no campo, do uso como hortaliça, especiaria e na medicina tradicional, sendo uma cultura agrícola de potencial socioambiental que atende a necessidade da agricultura familiar e comunidades em situação de risco e vulnerabilidade social. 
Esta planta caracteriza-se por apresentar hábito de crescimento prostrado, herbácea, anual, suculenta e flores amarelas e pequenas,não tolera locais encharcados e excesso de umidade (COELHO, 2010).

Pode ser consumida cozida, crua em saladas, podendo ser inserida na alimentação básica da família, inserindo pequenas quantidades no feijão, omelete, refogados e carnes. Com objetivo que a família adquira a segurança no consumo diário, e posteriormente produzir pratos mais elaborados para o consumo da beldroega, o que garante uma diversificação alimentar para a família.

A proposta de promover mudanças nos hábitos alimentares, resgatando as práticas alimentares locais, culturais e realizadas por povos antigos, plantas estas com alto teor nutricional, somente será possível com um processo educativo permanente e continuo, socializando e conscientizando a população do consumo das plantas alimentícias não convencionais (PANC), esclarecendo seus benefícios, vantagens e formas de consumo, através de receitas utilizadas no cotidiano familiar ou no preparo de farinhas.quem falou isso?

Esta pesquisa bibliográfica teve como objetivo resgatar as principais potencialidades de uso da Portulaca oleracea para promover a segurança alimentar em comunidade em situação de risco e vulnerabilidade social.

\section{RECEITA}

Uma boa pedida é o omelete de beldroega. Os ingredientes são: ramos de beldroega com folhas e flores, dois ovos, $1 / 2$ cebola picada, $10 \mathrm{ml}$ de azeite ou manteiga derretida, sal e pimenta calabresa. O modo de preparo consiste em refogar na manteiga ou no azeite a cebola e os ramos de beldroega picados ou inteiros, como preferir. Acrescentar os ovos batidos, o sal e a pimenta. Mexa levemente até o ponto desejado para o omelete. (PASSOS, 2018).

\section{MATERIAL E MÉTODOS}

A base metodológica é a abordagem crítica de caráter qualitativa. Trata-se de uma revisão de literatura sobre as potencialidades da beldroega, que possam auxiliar no processo de promoção da segurança alimentar em comunidade em situação de risco e vulnerabilidade social. Para discutir o trabalho foi elaborada uma pesquisa bibliográfica nas plataformas da Scielo, Google Acadêmico e periódicos da CAPES, com as palavras-chave: segurança alimentar; PANCS; Portulaca oleracea; em comunidade em situação de risco e vulnerabilidade social, beldroega; que possibilitaram debater a potencialidade da beldroega na segurança alimentar no benefício da comunidade. 


\section{RESULTADOS E DISCUSSÃO}

Constatou-se que o uso de plantas alimentícias não convencionais, ricas em nutrientes e minerais, como estratégia de complementação de alimentos, é apontado como uma alternativa para minimizar à situação de desnutrição e má alimentação mundial.

A beldroega é relatada como uma excelente fonte de compostos, principalmente gorduras, como o ômega-3 e ômega-6, ácidos orgânicos e compostos fenólicos, que apresentam alto poder antioxidante (OLIVEIRA et al., 2009). Contém saponinas (LU JR. \& PUTHETI, 2009) e flavonoides (ZHU et al. 2010).

As sementes de beldroega podem ser utilizadas para consumo humano, podendo extraír um óleo com uma boa relação entre ácidos gordos, que pode ser incorporado na alimentação humana (Stroescu et al., 2012).

A concentração de ácido alfa-linolénico nas folhas de beldroega é superior à encontrada em espinafre (Spinacia oleracea L.), mustarda (Brassica juncea L.), sorrel (Rumex acetosa L.) e beterraba (Beta vulgaris L.), sendo inferior à encontrada em diversas espécies de peixes, que são seres reconhecidos como fontes ricas neste ácido gordo (Simopoulos e Salem, 1986 citados por Palaniswamy et al., 2000). A beldroega é, reconhecidamente, a planta mais rica em ácidos gordurosos.

Tanto o ácido alfa-linolénico, como o ácido linoleico, não são sintetizados pelo Homem, mas permitem a síntese de outros elementos, como por exemplo as protagladinas (Palaniswamy et al., 2002), sendo esta síntese o seu principal benefício, uma vez que, estes elementos originados desempenham importantes funções em determinados órgãos do corpo humano (Lorgeril e Salen, 2004), o que mostra a importância do consumo de fontes destes ácidos gordos, que é encontrado em grande quantidade na beldroega.

Os flavonóides de $P$. oleracea L. são os constituintes biologicamente ativos, que mais têm sido descritos pela literatura, devido serem antioxidantes, antibacterianos, antivirais e anti-inflamatórios (Xu, 2006).

Segundo Odhav et al. (2007), a beldroega apresenta uma composição, por $100 \mathrm{~g}$ de peso seco, de $1361 \mathrm{mg}$ de cálcio, $333 \mathrm{mg}$ de fósforo, $148 \mathrm{mg}$ de sódio, $24 \mathrm{mg}$ de manganês, $3 \mathrm{mg}$ de cobre, $34 \mathrm{mg}$ de zinco, $1037 \mathrm{mg}$ de magnésio, e $42 \mathrm{mg}$ de ferro.

Em muitas partes do mundo ainda é considerada como "erva daninha com potencial teor nutricional" sendo descrita como um "alimento do futuro", devido ao seu elevado teor nutritivo e propriedades antioxidantes (Rinaldi, 2010). 


\section{CONCLUSÃO}

A partir desta pesquisa, fica clara a potencialidade de uso da beldroega na promoção da segurança e soberania alimentar em comunidades em situação de risco e vulnerabilidade social, pois, seu uso correto é uma alternativa de amenizar e evitar possíveis carências nutricionais, agindo como suplemento nutricional na alimentação.

Além de ser de uma planta de fácil cultivo e de crescimento rápido, tem boa adaptação às condições de clima tropical, o que facilita sua produção nas condições de agricultura familiar do extremo norte brasileiro.

Estudos que verifiquem as qualidades físico-química e biológica dos componentes comestíveis da beldroega e, os seus efeitos no consumo humano, ainda são necessários para avanço em pesquisas e aplicações com o vegetal.

\section{REFERÊNCIAS}

BOSI, G., GUARRERA, P. M.; RINALDI, R.; BANDINI-MAZZANTI, M. Ethnobotany of purslane (Portulaca oleracea L.) in Italy and morphobiometric analyses of seeds from archaeological sites in the Emilia Romagna Region (Northern Italy). Plants and Culture: seeds of the cultural heritage of Europe, Roma, p. 129-139, 2009.

COELHO, A. A. de O. P; GIULIETTI, A. M. O gênero Portulaca L. (Portulacaceae) no Brasil. Acta Bot. Bras., São Paulo, v. 24, n. 3, set. 2010.

DIAS, A. C. P.; PINTO, N. A. V. D.; YAMADA, L. T. P.; MENDES, K. L.; FERNANDES, A. G. Avaliação do consumo de hortaliças não convencionais pelos usuários das unidades do programa saúde da família (PSF) de Diamantina - MG. Alimentos e Nutrição, v. 16, n. 3, p. 279-84, 2005.

GALLINA, L. Representações sobre segurança alimentar e nutricional nos discursos de um Conselho de Alimentação Escolar. Saúde e sociedade, v. 21, n. 1, p. 89-102, 2012.

JESUS, Sofia Maria; Efeitos de extratos aquoso e etanólico de Portulaca oleracea L., sub-espécie sativa em Saccharomyces cerevisiae. Dissertação (Mestrado em Bioquímica) UNIVERSIDADE DE ÉVORA, Évora, 2017.

KINUPP, V. F.; LORENZI, H. Plantas Alimentícias Não Convencionais (PANC) no Brasil: guia de identificação, aspectos nutricionais e receitas ilustrdas. Nova Odessa: Ed. Plantarum, 2014.

Lorgeril, M.; Salen, P. 2004. Alpha-linolenic acid and coronary heart disease. Nutrition Metabolism e Cardiovascular Diseases, 14: 162-169. 
LU JR; PUTHETI, R. Compounds of Purslane extracts and effects of antikinetic fatigue. Journal Medicals, v. 3, p. 506-510, 2009.

MELÃO, I. B. Produtos sustentáveis na alimentação escolar: o PNAE no Paraná. Caderno IPARDES Instituto Paranaense de Desenvolvimento Econômico e Social, Estudos e Pesquisas, v. 2, n. 2 , 2012.

Odhav, B.; Beekrum, S.; Akula, Us.; Baijnath, H. 2007. Preliminary assessment of nutritional value of tradicional leafy vegetables in KwaZulu-Natal, South Africa. Journal of Food Composition and Analysus, 20: 430-435.

Oliveira, I. Valentão, P., Lopes, R. Andrade P., Bento, A. \& Pereira, J. (2009). Phytochemical characterization and radical scavenging activity of Portulaca oleracea L. leaves and stems. Microchemical Journal, 92, 129-134.

Palaniswamy, U.R.; Bible, B.B.; McAvoy, R.J. 2002. Effect of nitrate:ammonium nitrogen ratio on oxalate levels of purslane. A. Whipkey (eds). ASHS Press, Alexandria, VA, pág 453455.

Palaniswamy, U.R.; McAvoy, R.J.; Bible, B.B. 2000. Omega-3 fatty acid concentration in Portulaca oleracea is altered by nitrogen source in hydroponic solution. Journal of American Society Horticultural Science, 125: 190-194.

PASSOS, Mahedy Araujo Basttos. Panc é Pop: Plantas alimentícias Não convencionais em Roraima Listas de Espécies, aspectos gerais e receitas ilustradas. Boa Vista-RR: Ed. Folha de Boa Vista, 2018.

ROCHA, D. R. C.; PEREIRA JÚNIOR, G. A.; VIEIRA, G.; PANTOJA, L.; SANTOS, A. S.; PINTO, N. A. V. D. Noodles added of ora-pro-nóbis (Pereskia aculeata Miller) dehydrated. Alimentos e Nutrição, v. 19, n.4, p. 459-65, 2008.

SANTOS, J.V. Ricardo; Necessidades de azoto da beldroega (Portulaca oleracea Linn.) cultivada em substrato. Dissertação (Mestrado em Engenharia Agronómica) UNIVERSIDADE DE ÉVORA, Évora, 2014.

Simopoulos, A.P.; Norman, H.A.; Gillapsy, J.E.; Duke, J.A. 1992. Common purslane: A source of omega-3 fatty acids and antioxidants. Journal of the American College Nutrition, 11: 374-382.

Stroescu, M.; Stoica-Guzun, A.; Ghergu, S.; Chira, N.; Jipa, I. 2012. Optimization on fatty acids extraction from Portulaca oleracea seed using response surface methodology. Industrial Crops and Products, 43: 405-411. 
Revista Ambiente: Gestão e Desenvolvimento - Volume 11, n.01, Dezembro/2018

ISSN ONLINE:1981-4127

Stroescu, M.; Stoica-Guzun, A.; Ghergu, S.; Chira, N.; Jipa, I. 2012. Optimization on fatty acids extraction from Portulaca oleracea seed using response surface methodology. Industrial Crops and Products, 43: 405-411.

Xu, X., Yu, L. \& Chen, G. (2006). Determination of flavonoids in Portulaca oleracea L. by capillary electrophoresis with electrochemical detection. Journal of Pharmaceutical and Biomedical Analysis, 41, 493-499.

ZHU, Hongbin; WANG, Yuzhi; LIU, Yuxuan; XIA, Yalin; TANG, Tian. Analysis of Flavonoids in Portulaca oleracea L. by UV-Vis Spectrophotometry with Comparative Study on Different Extraction Technologies. Food analytical methods. v. 3, n. 2, p. 90-97, 2010. 